\title{
GCU
}

Glasgow Caledonian

University

University for the Common Good

\section{Rapid development of a low-cost web-based 360 virtual tour}

\author{
Insa-Iglesias, Maria; Jenkins, Mark D.; Morison, Gordon
}

Published in:

Proceedings of the 16th International Conference on Web Information Systems and Technologies

DOI:

$10.5220 / 0010176703150322$

Publication date:

2020

Document Version

Publisher's PDF, also known as Version of record

Link to publication in ResearchOnline

Citation for published version (Harvard):

Insa-Iglesias, M, Jenkins, MD \& Morison, G 2020, Rapid development of a low-cost web-based 360 virtual tour. in M Marchiori, F Domínguez Mayo \& J Filipe (eds), Proceedings of the 16th International Conference on Web Information Systems and Technologies. vol. 1, SciTePress, pp. 315-322, 16th International Conference on Web Information Systems and Technologies, 3/11/20. https://doi.org/10.5220/0010176703150322

\section{General rights}

Copyright and moral rights for the publications made accessible in the public portal are retained by the authors and/or other copyright owners and it is a condition of accessing publications that users recognise and abide by the legal requirements associated with these rights.

Take down policy

If you believe that this document breaches copyright please view our takedown policy at https://edshare.gcu.ac.uk/id/eprint/5179 for details of how to contact us. 


\title{
Rapid Development of a Low-cost Web-based 360 Virtual Tour
}

\author{
Maria Insa-Iglesias, Mark D. Jenkins and Gordon Morison \\ Department of Computing, Glasgow Caledonian University, Glasgow, U.K.
}

Keywords: 360 Virtual Tour, Open Source Code, Web-based Application.

\begin{abstract}
The use of 360-degrees Virtual Tour (VT) is a common practice in the education and tourism sector. It has recently gained popularity given the benefits of bringing physical spaces into a 360-degrees experience that can be explored in a simple and intuitive manner. Due to Covid-19, many Organisations wish to utilise immersive 360 technologies but many cannot afford it. In this paper, a 360-degree VT pipeline is proposed to allow Organisations to develop a VT that can compete in functionality with other sophisticated VT. Users with minimal coding experience are able to develop a low-cost web-based VT using a 360 camera, the Open Source tool Marzipano, the developed software framework and documentation from the GitHub repository. The contribution of this work is both the software framework, which complies with the Web Content Accessibility Guidelines (WCAG), for use with the Marzipano tool and a University case study along with a user evaluation to demonstrate the effectiveness of the approach. The usability evaluation run with the stakeholders demonstrates the acceptance of this 360 experience to allow new students to get a 360-degrees view of the GCU Glasgow Campus.
\end{abstract}

\section{INTRODUCTION}

The use of internet has exploded with the outbreak of Covid-19 and the business and education sector have moved face-to-face events into an online format. Like many other Universities in the UK, Glasgow Caledonian University (GCU) have joined online teaching platforms to run lectures and staff meetings, as well as, promotional events such as Open Days to recruit future student. Although Virtual Open Days are still a great option to explore what the University has to offer, students cannot experience a walk around the University facilities. As a result, many Universities have included Virtual Tour (VT) to their website providing a 360-degrees experience of a campus ${ }^{12} 3$.

$\mathrm{VT}$ is a trend in the education and tourism sector, bringing physical spaces into a virtual 3D environment that can be explored by a broad audience in a simple and intuitive manner. It is demonstrated that users tend to stay on the website longer when a VT is presented (Milano et al., 2011). Many Organisations have invested in the creation of web-based VT (Cho et al., 2002). It is demonstrated the the bene-

\footnotetext{
${ }^{1}$ https://uh.edu/campus-life/

${ }^{2}$ https://iviewd.com/gdgsignature/

${ }^{3}$ https://www.reading.ac.uk/VirtualTour/studentservices/tour/?indexstart=1
}

fits of 360-degree content in the tourism sector for evaluating your next holidays destination (Guttentag, 2010; Vishwakarma et al., 2020). Many Museums have also embraced VTs to offer a small taste of what is presented in the Museum before user visit it (Kabassi et al., 2019). Similarly, University VTs allow students to evaluate University destinations navigating through a 360-degrees experience of the teaching facilities, library, student rooms and shared open spaces (Shen et al., 2020). This can influence positively to student's application decision, making campus tour one of the best recruiting tools for prospective students (Mendolia-Moore, 2019).

Many Universities in the UK provide VT and video tours ${ }^{4}$. With the outbreak of Covid-19, VT have gained more popularity (Google Trends, 2012). Nevertheless, in uncertain times due to student numbers and other financial pressures, not all Universities can afford the cost to have a tour created or have the in house experience to have one developed, especially those Universities which have a large and spacious facilities. VT companies charge depending on the technology utilised to capture 360-degrees images, the VT features, and number of spaces required to capture. Finding a low-cost method so that Universities, $\mathrm{Mu}$ -

\footnotetext{
${ }^{4}$ https://www.ucas.com/undergraduate/what-andwhere-study/open-days-and-events/virtual-tours
} 
seums and other Organisations can build their own 360-degrees experience with minimal coding experience would be beneficial to many sectors.
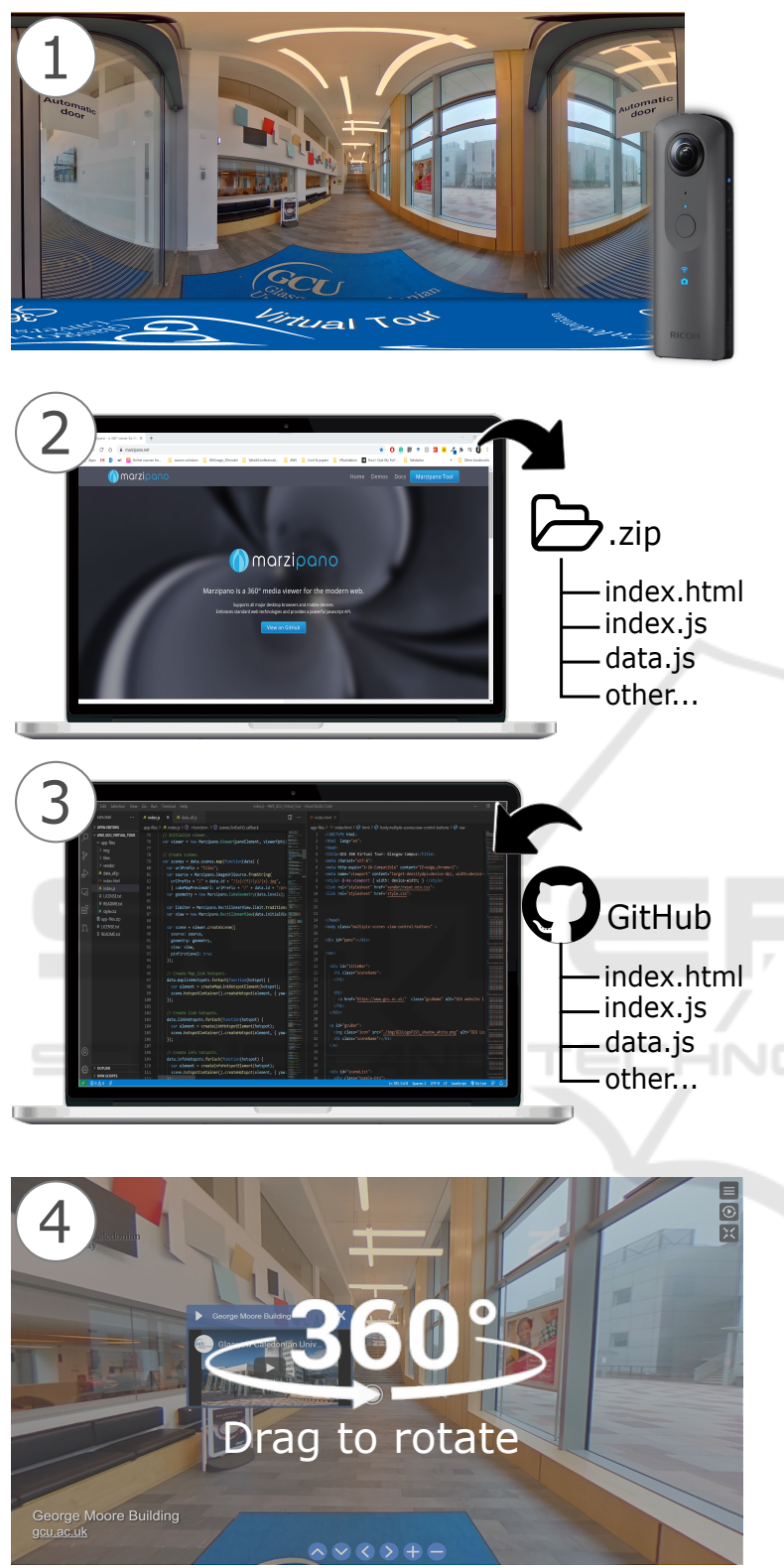

Figure 1: The Design and Development phase of the 360degrees VT pipeline proposed. This phases involves: the image acquisition and image editing (1); the web development that consists of uploading the 360 images to Marzipano Tool, export the web-application files (2), and then implementing the extra functionality copying the software framework developed (3); the publication of the VT using a web server.

This paper proposes a 360-degrees VT pipeline that allows users, who have no prior coding experience, developing a low-cost web-based $360 \mathrm{VT}$ that can compete with sophisticated VTs created by experts. The pipeline proposes guidance to develop a web-based 360 Virtual Tour using a JavaScript API called Marzipano and implementing the software framework published ${ }^{5}$ as part of the contribution. The HTML, JavaScript and CSS software framework developed integrates extra functionality to the VT proposed by Marzipano Tool by complying to the Web Content Accessibility Guidelines (WCAG) (level AA) (W3C, 2018). In addition, this papers introduces recommendations from the purchase of a 360 Camera to web hosting the VT. Following the method proposed, a case study is presented: "360 Virtual Tour of GCU - Glasgow Campus"6. This work enables Universities and other Organisations to stay competitive within the evolving landscape embracing $360 \mathrm{VT}$ technology (Mendolia-Moore, 2019).

The paper structure is as follow. Section 2 discusses the variety of options to develop a VT like paying a VT company, paying for an online course and the different open-source tools available. In addition, it introduces the challenges when developing a VT using an open-source tool and the contribution proposed in this paper. Section 3 presents the 360-degrees VT pipeline that will allow developing a low-cost web-based VT with minimal coding experience. It includes the software framework proposed in this work so that users can implement them in their VT project and acquire extra functionality like a map page and a variety of hotspots classes. A case study of GCU Glasgow Campus is presented (Section 4), which demonstrates the applicability of the 360degrees VT pipeline proposed. Furthermore, a usability evaluation was developed by the team and University students (Section 5). Finally, the paper concludes with a conclusion and future work section (Section 6).

\section{RELEVANT WORK}

Many companies are dedicated to developing VTs (3DVista , 2015) (iViewd, 2017) (Panoskin, 2019). Other companies provide online courses (Virtual Tour Pro, 2019) (Udemy, 2015). These services can be very expensive and may not meet the client requirement (Ceulemans et al., 2018).

360 VTs can be developed in a cheaper manner and customised to client requirement using tools like Google VR Tour Creator (Google, 2018) and Pannellum (Pannellum, 2015), although those present limited functionality to compete with the most soph-

\footnotetext{
${ }^{5}$ https://github.com/Insa-Maria/Virtual_Tour.git

${ }^{6} \mathrm{http} / / /$ campus.gcu.ac.uk/
} 
isticated 360 VT. These deliver additional functionality paying extra packages. Tools like kprano (krpano, 2020), Matterport (Matterport, 2020) and Roundme (Roundme, 2016) allow testing the functionality through a temporary free trial package, however purchasing a license is required to be able to develop and share a VT.

To our best knowledge, Marzipano ${ }^{7}$ is the only Open Source tool that allows the design, development and customisation of VTs, although it requires programming skills in JavaScript, HTML and CSS. Marzipano also provides demos which show the possible functionality that can be developed by who are experienced in JavaScript and Marzipano Tool. It is true that forums like Quora (Quora, 2010) and Google Groups (Google Groups, 2017) support experienced and nonexperienced users when encounter coding errors, for example. However, these forums are used to answer specific errors rather than providing detailed guidance from start to end on your project. Given the capability of Marzipano Tool, this work aims at proposing an Open Source pipeline to develop and custom a lowcost web-based VT with minimal coding experience using Marzipano and a 360 camera.

There is work that describes the stages to develop a 360-degrees experience, yet this work focused on the usage of non Open Source software increasing the overall costs of the approach. For example, these works (Collier III et al., 2017; Wessels et al., 2014) describe the phases to develop a 3D VT for cultural heritage industry using different technology from 360 Cameras. These other work (Argyriou et al., 2020; Ceulemans et al., 2018) propose a set of requirements and design considerations for 360 immersive video. Although these guidelines are beneficial when exploring the VTs capability, it does not assist nonexpert on the development of a VT. This work aims at providing a software framework and full documentation to allow users to rapidly develop their VTs.

\section{VIRTUAL TOUR PIPELINE}

As mentioned before, there are many approcahes to develop a low-cost web-based VT (Section 1) given the software, image acquisition technology and delivery choice to share the VT, among others. The 360degrees VT pipeline proposed is designed for those Organisations that are willing to:

1. Purchase a 360 Camera to capture 360 images/videos.

2. Develop a Web-based application to share the VT.
3. Utilise Marzipano Open Source tool to develop VT.

This work aims at proposing a methodology to assist those Organisations, which accomplish the above requirements, developing a low-cost web-based VT. Being able to develop its own VT will allow Organisations to update it at any time at no extra cost and utilise the 360 camera for other activities like live streaming an event in a 360-degrees experience. The 360degrees VT pipeline proposed consists of the following phases (see Figure 4): Research, Planning, Design and Development, Hosting and Feedback. The last three phases are iterated until obtaining the desired web-application.

\subsection{Research}

The only associated cost with the development of the VT is the purchase of the camera. The camera choice depends on the budget and specification required. Nowadays, there is a wide variety of 360 camera, which prices range between $£ 250$ - $£ 45,000$ (Ceulemans et al., 2018). Although there are lowpriced 360 cameras, it is important to consider the image quality as a persuasion value. The higher-quality content is shown in a 360-degrees experience, the better telepresence is perceived by users in a virtual environment and therefore more persuasion.

Apart from checking the camera features, the following specifications will allow a better performance during the image acquisition phase. Battery life and charging time are two key specifications, especially for those Organisations with large and spacious facilities. A longer battery life will allow longer period capturing images before charging the camera, which could two times longer longer than the period capturing 360 images.

There are camera extras that are highly recommended to purchase to ease the development of a high-quality VT. These are the tripods and monopods. There are many options depending on the height, base size, versatility to use it on different ground types (e.g. road, grass, stairs), and image acquisition location (e.g. indoors, outdoors or windy outdoors locations). It is important to consider that the smaller the tripod base is, the better hidden the tripod will be in the 360 images. Having the base of a 360 image covered by the black frame of the tripod can be disturbing. The Image Editing phase (Section 3.3.2) will cover some tips how to negate this issue.

\footnotetext{
${ }^{7}$ https://www.Marzipano.net/
} 


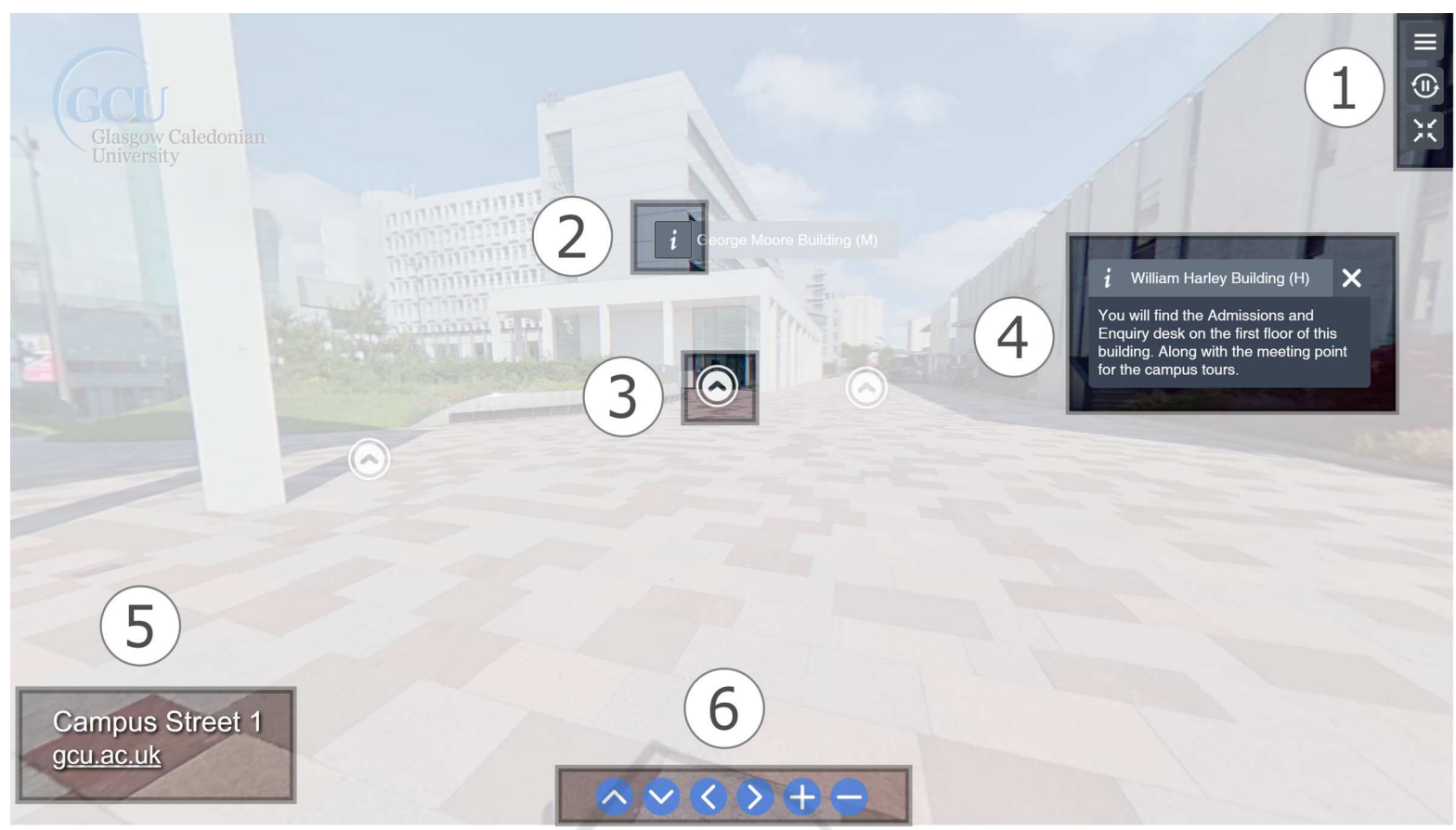

Figure 2: The 360 VT of GCU: Interface Design. This figure shows the web interface design. The control buttons (1) are situated at the top right that consist of the menu button (top), rotation button (middle) and fullscreen button (bottom). Clicking the menu button the drop down menu is displayed on the right side of the screen (see Figure 3). Clicking the informative hotspot (2), an informative panel is displayed (4). Clicking on the link hotspot (3), users explore more University facilities. The bottom left title (5) is the name of the installation being explored by the user. The link button The control view buttons (6) are situated at the centre bottom of the screen and clicking on them users can rotate to the side to control the view.

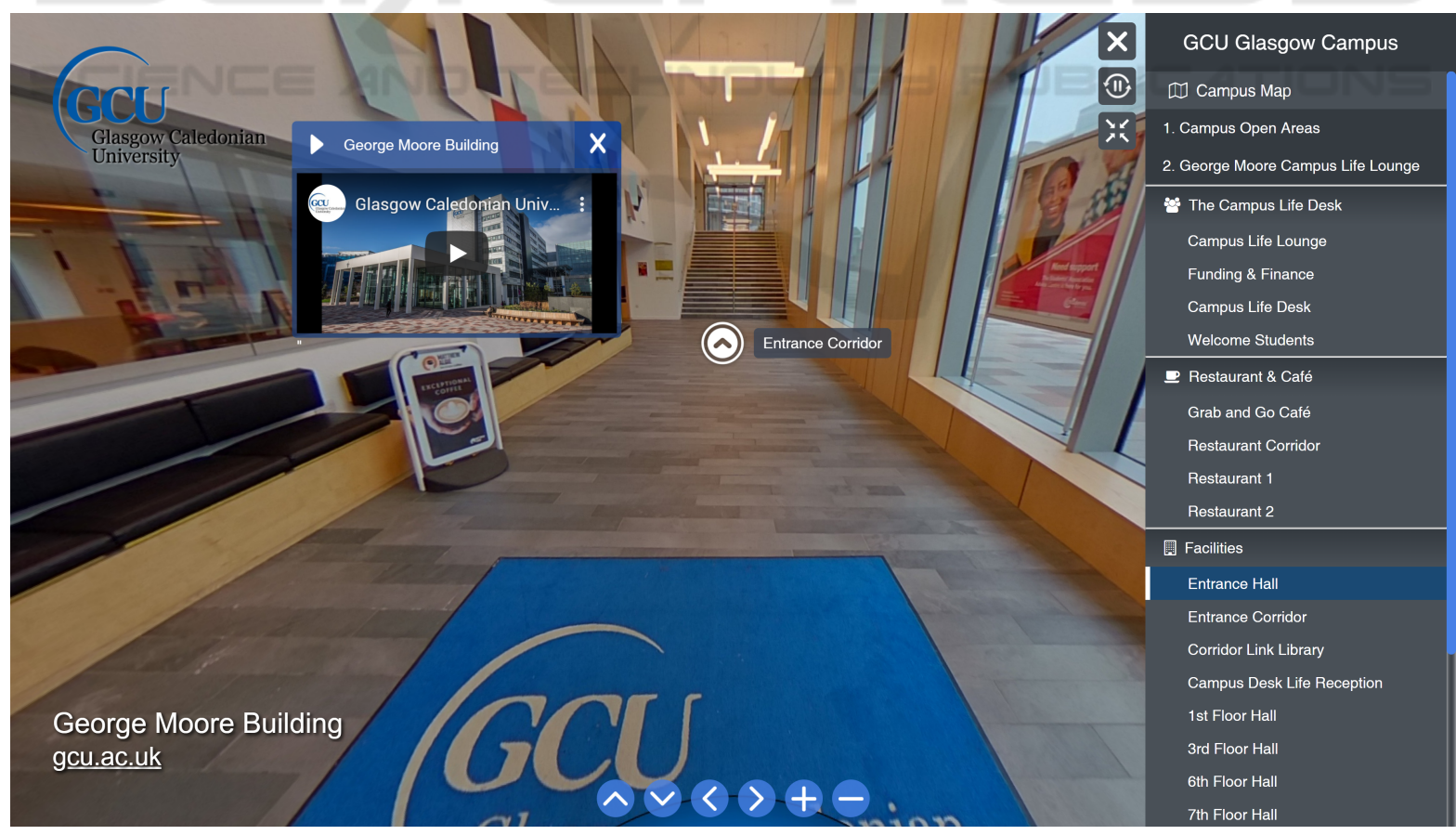

Figure 3: The 360 VT of GCU: George Moore Building. This figure shows the 360-degrees experience that users have when entering to George Moore Building. When clicking the video hotspot (play icon coloured in blue), multimedia content is played. Clicking the link hotspot (white arrow icon), users travel to the Entrance Corridor to explore more facilities. 


\subsection{Planning}

The second step consists of planning the image acquisition phase, which will optimise the time spent filming all facilities. The planning consists of creating a detailed list of all facilities to be captured and specify the following timings: walking to the facility, room set up, filming time and room closure. Depending on the dimension and location of the facilities, timings will change considerably. Table 1 shows a schedule reference when setting times for standardsize facilities like teaching labs (Case A), and for bigsize facilities like a sports and library hall (Case B).

Such a plan allows the uses to calculate approximately the usage time of the camera, and therefore estimate the number of facilities able to film before the camera's battery finished. This planning allows for development of an approximate schedule with the enter and exit time to each facility.

Table 1: This table shows the timings considered to approximate the time needed to film the GCU facilities.

\begin{tabular}{ccc}
\hline Task & Case A (min) & Case B (min) \\
\hline \hline Walk to facilities & 5 & 10 \\
Set up & 10 & 20 \\
Image Acquisition & 5 & 15 \\
Closure & 10 & 10 \\
\hline Total & $30 \mathrm{~min}$ & $55 \mathrm{~min}$ \\
\hline \hline
\end{tabular}

\subsection{Design and Development}

This phase comprises (see Figure 1) the Image Acquisition, Image Editing and Web Development.

\subsubsection{Image Acquisition}

This phase consists of capturing 360 images of the facilities required following the schedule established. The following considerations will enhance user's telepresence in a room: positioning the camera at the center of the facility, setting the camera height at the human eyes height, and facing the front lens towards the point of view you would like users to start exploring (although it is also recommend to face the camera lens toward the wall at a close distance for a better images stitching).

\subsubsection{Image Editing}

This phase consists of editing the 360 images to remove the tripod black frame that appears at the bottom of most of the 360 images. There are many tech- niques to remove it, for example adding a logo, bluring the image or blending it in the image (see Figure 1 (1)). It is highly recommended to organise the images in folders, for example, grouping images by buildings or floors, and rename files sequentially. This way, when 360 images are uploaded to Marzipano Tool, these will be uploaded in alphabetically order and therefore organised by the groups established.

\subsubsection{Web Development}

This phase consists of uploading the 360 images into Marzipano Tool. This tool allows anyone to upload unlimited 360 images, which are processed and added into a basic web application that is exported as a .zip folder with the following files inside a folder called "app-files": data.js, index.html, index.js and style.css (see Figure 1 (2). The resulted web application has a limited functionality, but implementing the code developed in JavaScript, HTML and CSS (see Figure 1 (3), the tour can be transformed into a sophisticated VT like the one shown in Figure 3 (read Section 4).

\subsection{Hosting}

The hosting phase consists of finding the most suitable web hosting service to upload the web application files to a server, set a domain and share the web link to enjoy the 360-degrees experience (see Figure 1 (4)). There are many manners to web hosting a VT using (a) a traditional method where you pay for a fixed monthly fee (e.g. Hostgator ${ }^{8}$ or Blue.host ${ }^{9}$ ) or (b) a modern method where you pay for what you use, in others, you pay depending on the web files size, the number of visits to your website and other variables (e.g. Amazon Web Services $(\mathrm{AWS})^{10}$ ).

\subsection{Feedback}

The final step consists of evaluating the web-based VT. This involved checking that the VT requirements established by the Organisations are met, ensuring that the website complies with the the WCAG (level AA) (W3C, 2018) making the web content accessible to wide range of audiences including those with disabilities.

\footnotetext{
${ }^{8}$ https://www.hostgator.com/web-hosting

${ }^{9} \mathrm{https}: / / \mathrm{www}$. bluehost.com/hosting

${ }^{10} \mathrm{https}$ ///aws.amazon.com/
} 


\begin{tabular}{l|l|l|l|l|} 
& Week 1 & Week 2 & Week 3 & Week 4 \\
\hline Research & & & & \\
\hline Planning & & & & \\
\hline Image Acquisition & & & & \\
\hline Image Editing & & & & \\
\hline Web Development & & & & \\
\hline Hosting & & & & \\
\hline Feedback & & & & \\
\hline
\end{tabular}

Figure 4: The Gant Diagram. The case study in the 360 VT of GCU was developed in four weeks following the proposed 360-degrees VT pipeline.

\section{VIRTUAL TOUR OF GCU}

This section presents "360 Virtual Tour of GCU Glasgow Campus", a case study that demonstrates the applicability of the 360-degrees VT pipeline proposed.

With the Covid-19 outbreak, GCU's Department of Computing reassessed the goals for the academic year and designed a plan to invest in a 360 camera to develop a low-cost web-based VT. The GCU 360 VT was developed in four weeks (see Figure 4), producing a 360-degrees experience that allows future students to navigate and explore the facilities of the Glasgow campus, thus helping students to gain a better perspective of the campus in these times where Open Days are online.

The 360 camera utilised in this case study was a Ricoh Theta V along with an Insta360 One X invisible selfie stick and a mini tripod to mount the selfie stick. Several online meetings with "Marketing and Recruitment" manager and GCU Schools head of department were run until the final list of facilities required to film was agreed. In addition, the following functional requirements were agreed:

- The tool should provide a Campus Map

- The tool should guide students from the Campus entrance to the main facilities

- The tool should allow users navigate into the main facilities and interact with elements such as informative panels and videos

In this time of social-distancing measures, planning the image acquisition schedule was essential to inform Security Team when and for how long it was required accessing to each building. This allow us to obtain access to GCU earlier than expected. In four days with shifts of six hours every day including two hours to charge the camera's battery, the team capture 360 images of around 140 environments distributed along 9 buildings.

The images were edited adding GCU logo at the bottom of all 360 images, renamed and organised by Campus buildings. After uploading the files in Marzipano Tool and exporting the web application files, the software framework proposed in this paper was implemented to acquire the additional functionality:

- Campus map: this map (see Figure 5) provides an overview of the Glasgow Campus buildings and Campus entrances. Implementing new code lines to the index.html, index.js and data.js, a new class of hotspot was acquired to link the map with the building entrance when clicking on the building hotspot.

- Video Hotspots: the basic functionality proposed in Marzipano Tool consists of two type of interactive hotspot: an informative hotspot and a link hotspot to navigate to the following 360 images (see Figure 2 (2) and (3) respectively). In addition to the building hotspot class, the code developed in index.js and data.js files introduces a video hotspot class that displays a multimedia content relevant to the environment along many 360 images.

- Accessible Dropdown Menu: the vertical menu proposed by Marzipano is a single level list and do not provide the guidelines to group 360 images, especially when VTs have large number of images. Given that the 360 VT of GCU included around 140 images (an image per room except those large rooms like the library where 2 images were taken), the code proposed in index.html introduces a solution to this complying the WCAG standards.

The team decided to use a web server for webhosting the VT as a static website using Amazon Simple Storage Service (Amazon S3).

\section{USER EVALUATION}

Members from the "Marketing and Recruitment", the "Student Engagement and Communications" and "School of Computing" Department evaluate the usability of the VT. A total of 8 people (4 GCU students and 4 GCU staff members) participated. Due to Covid-19, the interview was conducted via videocall.

Each participant joined the videocall individually. The study consisted of an initial description of 360 immersive technologies, followed by a testing of the web-based VT of GCU. The link ${ }^{11}$ was shared via the

\footnotetext{
${ }^{11} \mathrm{http}: / /$ campus.gcu.ac.uk/
} 


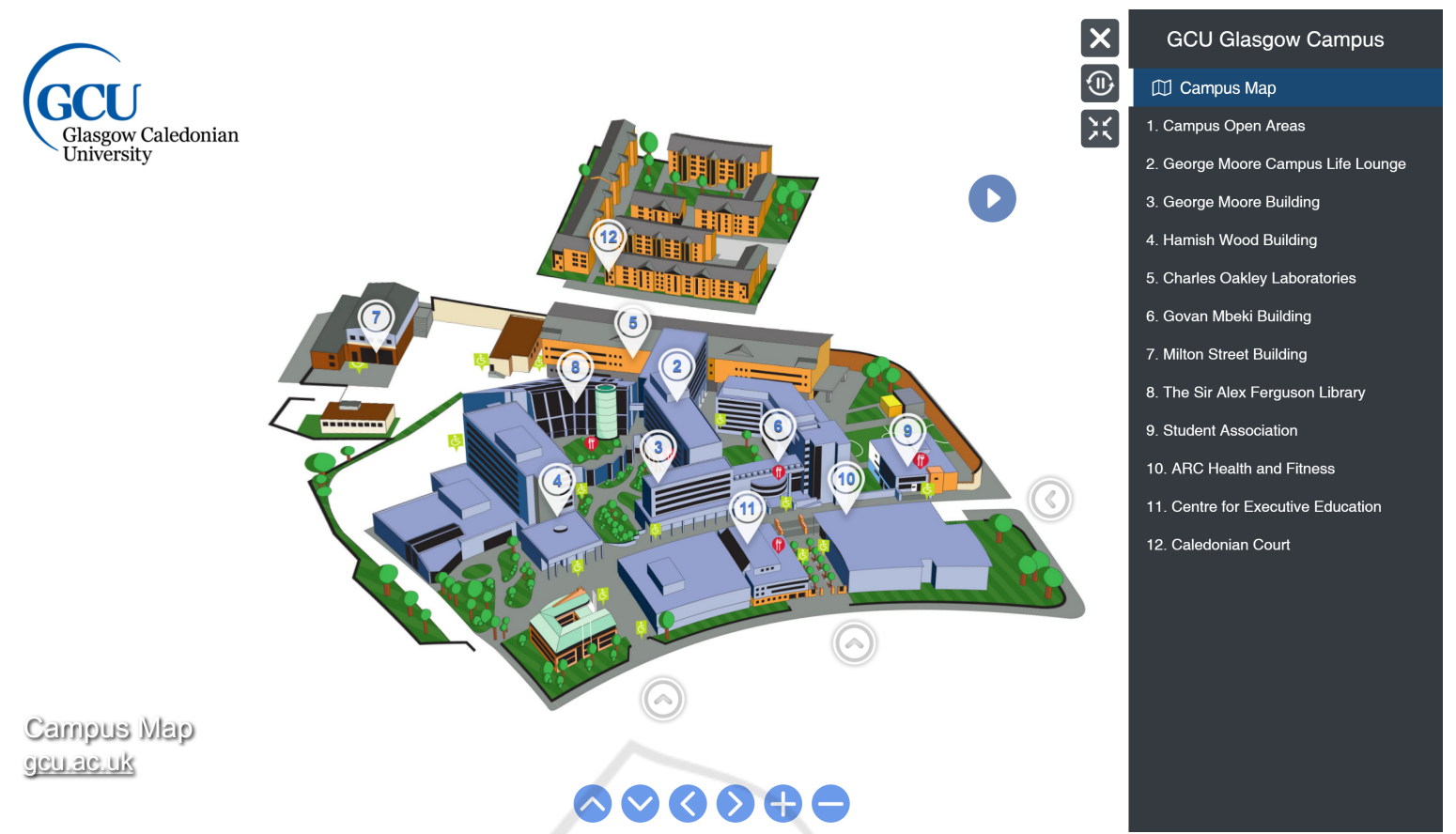

Figure 5: The 60 VT of GCU: Campus Map. This figure shows the main Campus entrances and building marked with an interactive arrow and location icon, respectively. Clicking on the icon, users navigate through the facilities simulating what it would be a Campus walking tour. This map page allows users go back once they finish exploring a building.

videocall chat and they had 10 minutes to explore the 360 experience. After, participants required to discuss the good and bad practices when utilising the webbased VT interface.

The feedback delivered was very positive by all participants. They saw the positive impact of the webbased VT to allow new students to get a 360 view of our GCU campus, roam around the library building, classrooms and main social spaces. "Overall I think it looks brilliant!" and "I found it easy to navigate! I like that when you select a building from the drop down menu, it allows you to select different areas that are found there".

Nevertheless, they identified points of improvement. Some students were confused when trying to return to the Campus Map after exploring a building: "I was a bit confused about how to return to the map(...) it would be slightly easier to have a click link back to the map rather than having to go into the three lines at the top and get back to the map that way". They suggested that having a button similarly to the fullscreen button would be helpful to back to the Campus Map "rather than having to go into the three lines at the top and get back to the map that way".

GCU staff members suggested as future work the need to make the web-based VT accessible using different devices, such as phones. Although it works in a wide variety of browsers and devices, some function- ality is limited in smartphones. One of the students suggested: "It would be good to say what's in each of the buildings like for example where it's got "George Moore Building" having like "Campus Life Desk" because realistically students are not just going to know it". Although this information is covered through the 360 images of Campus Open Areas, adding informative panels describing the main facilities in each building in the Campus Map page would cover such suggestion.

\section{RESULTS AND FUTURE WORK}

The contribution presented is a 360-degrees VT pipeline that allows Organisations such as Universities to quickly develop a low-cost web-based VT, just investing in purchasing a 360 camera and paying a monthly fee for a web hosting the VT. The case study "360 Virtual Tour of GCU - Glasgow Campus" demonstrates the applicability of the pipeline. This will enable many Universities to provide a 360degrees experience of a Campus visit, similarly to face-to-face Open Days, and being at the same level to their competitors.

The future work of this project involves the development of a instruction in the GitHub repository. Such GitHub repository will provide a detailed de- 
scription of pipeline phases, including: (i) the comparison table of 360 camera ranked by specifications to assist users on the camera choice, (ii) the detailed schedule utilised during GCU image acquisition phase, (iii) the tutorials followed to edit 360 images, (iv) a step-by-step of the code edited to obtain a complete VT functionality, (v) tips for selecting the most appropriate web hosting service and (vi) feedback provided by the team. In addition, the GitHub repository will be helpful for those Organisations who need support following the pipeline proposed. To demonstrate the effectiveness of the pipeline, a usability study to evaluate the effectiveness of the webbased instructions to develop a low-cost web-based VT will also be included.

\section{REFERENCES}

3DVista (2015). Virtual tours, $360^{\circ}$ video and vr software. https://www.3dvista.com/ Last accessed on 2020-0828.

Argyriou, L., Economou, D., and Bouki, V. (2020). Design methodology for 360 immersive video applications: the case study of a cultural heritage virtual tour. Personal and Ubiquitous Computing, pages 1-17.

Ceulemans, C., Klaassen, R. G., and Kreuk, M. M. d. (2018). 360-degree virtual tour for educational purposes. an exploration on the design considerations and decisions.

Cho, Y.-H., Wang, Y., and Fesenmaier, D. R. (2002). Searching for experiences: The web-based virtual tour in tourism marketing. Journal of Travel \& Tourism Marketing, 12(4):1-17.

Collier III, J. P., Lewis, J. W., and Bernard, B. P. (2017). Using photogrammetry to create a virtual campus tour for the htc vive.

Google (2018). Tour creator. https://arvr.google.com/ tourcreator/ Last accessed on 2020-08-28.

Google Groups (2017). Marzipano google groups. hhttps: //groups.google.com/forum/\#!forum/marzipano Last accessed on 2020-08-28.

Google Trends (2012). Google trends: Virtual tour. https://trends.google.com/trends/explore?q= virtual $\backslash \% 20$ tour Last accessed on 2020-08-28.

Guttentag, D. A. (2010). Virtual reality: Applications and implications for tourism. Tourism management, 31(5):637-651.

iViewd (2017). iviewd technologies. https://iviewd.com/ Last accessed on 2020-08-28.

Kabassi, K., Amelio, A., Komianos, V., and Oikonomou, K. (2019). Evaluating museum virtual tours: The case study of italy. Information, 10(11):351.

krpano (2020). krpano panorama viewer. https://krpano. com/ Last accessed on 2020-08-28.

Matterport (2020). Capture, share, and collaborate the built world in 3d. https://matterport.com/ Last accessed on 2020-08-28.
Mendolia-Moore, T. (2019). The college path: A virtual tour.

Milano, R., Baggio, R., and Piattelli, R. (2011). The effects of online social media on tourism websites. In ENTER, pages 471-483. Citeseer.

Pannellum (2015). A lightweight panorama viewer for the web. https://pannellum.org/ Last accessed on 202008-28.

Panoskin (2019). Best virtual tour software - free virtual tour app. https://www.panoskin.com/ Last accessed on 2020-08-28.

Quora (2010). A place to share knowledge and better understand the worldision impairment and blindness. https: //www.quora.com/ Last accessed on 2020-08-28.

Roundme (2016). Virtual tours made simple. https:// roundme.com/ Last accessed on 2020-08-28.

Shen, J., Wang, Y., Chen, C., Nelson, M. R., and Yao, M. Z. (2020). Using virtual reality to promote the university brand: When do telepresence and system immersion matter? Journal of Marketing Communications, 26(4):362-393.

Udemy (2015). 360 panoramic photography for virtual tours and object vr. https://www.udemy.com/course/ panoramic-photography/ Last accessed on 2020-0828.

Virtual Tour Pro (2019). Learn how to plan, shoot, edit, get clients and get paid for your virtual tours. https: //www.virtualtourpro.com/ Last accessed on 2020-0828.

Vishwakarma, P., Mukherjee, S., and Datta, B. (2020). Travelers' intention to adopt virtual reality: A consumer value perspective. Journal of Destination Marketing \& Management, 17:100456.

W3C (2018). Web content accessibility guidelines (wcag) 2 level aa conformance. https://www.w3.org/WAI/ Last accessed on 2020-08-29.

Wessels, S., Ruther, H., Bhurtha, R., and Schroeder, R. (2014). Design and creation of a $3 d$ virtual tour of the world heritage site of petra, jordan. Proceedings of AfricaGeo, pages 1-3. 